\title{
Social disadvantage, family composition, and diabetes mellitus: prevalence and outcome
}

\author{
J H Baumer, L P Hunt, J P H Shield
}

\begin{abstract}
Objective-To investigate the relation between social disadvantage and family composition on diabetes prevalence and diabetes care outcome.

Design-Retrospective audit in the south west of England of 801 children with diabetes mellitus.

Main outcome measures-Prevalence of diabetes in relation to the Townsend index. Admissions to hospital with diabetes related problems, glycated haemoglobin, time spent in hospital, outpatient attendance rates.

Results-There was no association between social status and diabetes prevalence. Social deprivation increased the likelihood of admission for hypoglycaemia. Children living with a single parent were more likely to be admitted to hospital with a diabetes related problem and stay in hospital longer. Having either a parent with diabetes or a single parent increased the rates of clinic nonattendance. No association was identified between medium term diabetes control and either social disadvantage or single parent status.
\end{abstract}

Conclusions-Social disadvantage has no effect on diabetes prevalence and little on diabetes outcome in childhood. Family structure and parental diabetes have adverse effects on some aspects of diabetes outcome.

(Arch Dis Child 1998;79:427-430)

Keywords: diabetes mellitus; social deprivation; family structure

The reason for the observed increase in incidence of type I diabetes is currently the subject of considerable research interest. The rapid changes seen suggest environmental as opposed to genetic explanations for the increase. The finding of associations between social circumstances (such as household overcrowding) and the prevalence of diabetes mellitus provides strong supportive evidence for the role of environmental factors in disease aetiology. ${ }^{1}$ A recent regionwide audit of childhood diabetes in the south west region of the UK provided an opportunity to examine the association between social deprivation and the prevalence of diabetes as well as outcomes of diabetes care. ${ }^{2}$

Various people have reported a possible association between the aetiology of childhood diabetes and social deprivation. Most reports have found a stronger association with increas- ing social advantage, ${ }^{3-7}$ although others have reported an increased risk with mothers having a lower educational level, with fathers in manual occupations, ${ }^{89}$ and with increasing level of deprivation, ${ }^{10}$ and yet others have found no association with social class. ${ }^{11}$ However, there are important differences in study methodology between existing reports on social deprivation in insulin dependent diabetes mellitus. Some have reported the relation between the prevalence of childhood diabetes and social disadvantage ${ }^{3-5}$ whereas others have compared the incidence. ${ }^{6-11}$ Measures of social advantage have included socioeconomic group,,$^{3-5} 81011$ household income, ${ }^{6}$ Carstairs' index ${ }^{12}$ of deprivation, ${ }^{7}$ mother's level of education, ${ }^{8}$ and Townsend's deprivation index. ${ }^{9}{ }^{13}$ The age range of children studied has varied, and in some studies the index families have been compared with the whole population, including all age ranges. ${ }^{367}$ In one study, ${ }^{5}$ only 18 children were ascertained, and in another, ${ }^{3}$ $<100$ children with diabetes were included. Two studies involved children presenting 20 to 30 years ago. ${ }^{511}$

In addition to any possible causal association, deprivation and psychological factors such as parental diabetes may influence diabetes outcome. Few studies have been conducted in this area but there are indications that parental diabetes can have an unexpectedly detrimental effect on diabetes outcome in childhood, ${ }^{14}$ as can social and economic deprivation in adults with insulin dependent diabetes. ${ }^{15}$

\section{Methods}

As part of a regional audit of children with diabetes, all children and young people with diabetes mellitus living in the south west of England in 1994 were identified through contact with the diabetes teams caring for them. ${ }^{2}$ Measures of outcome included glycated haemoglobin results, admissions to hospital associated with diabetes and at diagnosis, clinic attendance rates, clinic contacts with a dietitian, clinic measurements of height and weight, screening rates for hypertension, microalbuminuria, and retinopathy. Associations with service structure including consultant and diabetes nurse caseload and the existence of diabetes clinics were investigated. Information was collected for each child on their family composition, whether either parent had diabetes, and their postcode.

Each child's postcode was used to calculate a Townsend deprivation index. ${ }^{13}$ From each child's postcode the corresponding enumeration district is mapped, then the following four 
Table 1 Numbers of children by age at diagnosis, at 31 December 1994, and by duration of diabetes

\begin{tabular}{lrrrr}
\hline \multicolumn{4}{l}{ Age (years) } & \\
\cline { 2 - 5 } & $<5$ & $5-9.9$ & $10-15.9$ & $\geqslant 16$ \\
\hline Number at diagnosis $(\mathrm{n}=761)$ & 274 & 280 & 205 & 2 \\
Number at 31 December 1994 $(\mathrm{n}=801)$ & 45 & 173 & 411 & 172 \\
Duration of diabetes (number of children) & 439 & 242 & 102 & 10 \\
\hline
\end{tabular}

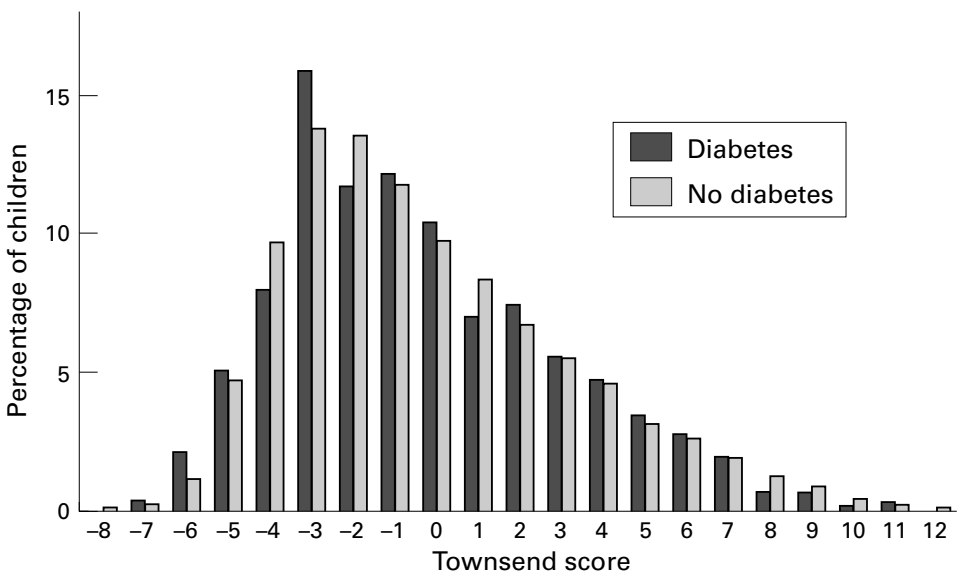

Figure 1 Townsend scores of children with and without diabetes.

variables from the 1991 census information for that enumeration district are used:

(1) The percentage of households without access to a car (a proxy indicator of income)

(2) The percentage of households not in owner occupied accommodation (a proxy indicator of wealth)

(3) The percentage of households in overcrowded accommodation - that is, more than one person in each room (a direct indicator of material living conditions)

(4) Unemployment as a percentage of the commercially active population (a measure of general lack of material resources and insecurity).

A $\mathrm{z}$ score (with a maximum of 3 and a minimum of -3) is calculated for each variable (which is standardised to have a mean of 0 and a standard deviation of 1 for all the district authorities of England). These scores are then added together to create the Townsend deprivation index for the enumeration district, with a maximum of 12 for the most deprived and a minimum of -12 for the least deprived.

Townsend indices were obtained for all children in south west England who were $<16$ years at the time of the 1991 census. The children $<16$ years old with diabetes were subtracted from this population to estimate the distribution of Townsend scores for children without diabetes; the scores for the children with and without diabetes were compared.

Associations were investigated between three social variables (Townsend indices, whether the child was not living with both parents, and whether either parent had diabetes) and a series of outcome measures. A full list of the outcomes studied in the audit and a detailed description of the statistical methods have been described previously. ${ }^{2}$ The three social vari- ables listed were considered as "predictors", first individually and then together with the child's age, duration of diabetes, and the dose of insulin (units $/ \mathrm{kg}$ ) using backward deletion of non-significant variables in a series of logistic regression models. The consultant care group ("specialist", "non-specialist", or "tertiary") was included if this was significant over and above these other factors. ${ }^{2}$

\section{Results}

Table 1 shows the demographic characteristics of the children with diabetes in the regional audit.

Townsend indices were available for 766 out of 801 of the children with diabetes in the audit (median -0.58 , range -6.28 to 11.73 ). A quarter of the children $(193 / 785$ or $24.6 \%)$ did not live with both parents and 10\% (78/775) had a parent with diabetes.

Figure 1 shows the frequency distribution of Townsend indices for the 617 children under the age of 16 for whom a Townsend index within the region was available, compared with indices for the estimated 689514 children without diabetes in the region. There was no significant difference between these two distributions (two sample Kolmogorov-Smirnov tests, $\mathrm{p}>0.10)$.

Of the 193 children not living with both parents, 164 were apart from their fathers, 16 from their mothers, and 13 from both parents. In $76 \%$ of the families with one parent absent the remaining parent was unsupported by a partner. Children living with both parents had significantly lower Townsend scores than the remainder (median -0.87 , range -6.28 to 11.73 compared with 0.045 , range -5.33 to $11.73, p=0.009$ Mann-Whitney U test).

Forty nine children had a father with diabetes and 37 a mother with diabetes. The Townsend scores of children of parents with diabetes were not significantly different from the remainder. There was no association between parental diabetes and separation.

We originally reported that $4.1 \%$ of children seen over the complete 12 month interval ( $n=677$ ) were admitted at any time for hypoglycaemia. The percentage was increased significantly in children with higher Townsend indices $(\mathrm{p}=0.029 ; \mathrm{n}=658)$; the estimated rate for the mean index (0) was $3.8 \%$, with rates approximately doubling as the index increased by six points (odds ratio (OR) 2.06; $95 \%$ CI 1.10 to 3.87 ). This was confirmed after adjustment for other significant variables: age, dose of insulin, and consultant group ( $p=0.031, n=635$, OR for each six point increase in index $2.18,95 \%$ CI 1.09 to 4.38 ).

Similarly, $7.1 \%$ of children were admitted with ketoacidosis or for stabilisation over the 12 month period $(n=675)$. Admission was significantly more likely for children not living with both parents $(11.9 \%$ compared with $5.8 \%, \mathrm{p}=0.013, \mathrm{n}=663)$ and if the child had a parent with diabetes $(14.5 \%$ compared with $6.4 \%, p=0.035, n=652)$. Adjustment for each other as well as insulin dose confirmed the former, but the latter was of borderline significance $(p=0.015$, OR 2.24, 95\% CI 1.19 to 
$4.21 ; \mathrm{p}=0.068$, OR $2.29,95 \%$ CI 1.00 to 5.23, respectively, $n=625)$. The percentage of children admitted was unrelated to the Townsend index.

Taking the above together, $9.8 \%$ of children were admitted for any diabetes related problem over a complete 12 month interval $(n=674)$. The percentage was increased significantly in children not living with both parents $(15.7 \%$ compared with $8.2 \%, \mathrm{p}=0.008, \mathrm{n}=662)$ and in those with a parent with diabetes $(19.4 \%$ compared with $9.0 \%, \mathrm{p}=0.018, \mathrm{n}=651)$. Adjustment for other significant variables (age and dose of insulin) confirmed the former association but the latter was only of borderline significance $(\mathrm{p}<0.001$, OR $2.23,95 \%$ CI 1.27 to $3.92 ; \mathrm{p}=0.063$, OR $2.09,95 \%$ CI 1.00 to 4.38 , respectively, $\mathrm{n}=624$ ).

Children not living with both parents had significantly more days each month in hospital for diabetes related problems (mean 0.115 days each month $(\mathrm{n}=192) \quad v 0.025$ days each month $(n=589), p=0.001$ Mann-Whitney U test).

Pre-planned outpatient appointments were less likely to have been kept in the case of children who did not live with both parents $(78.8 \%$ of individual appointments compared with $83.7 \%, \mathrm{p}<0.001, \mathrm{n}=765)$ and if the child had a parent with diabetes $(77.9 \%$ compared with $82.5 \%, \mathrm{p}=0.061, \mathrm{n}=755)$. These were both statistically significant after adjustment for each other and for other significant variables - that is, diabetes and consultant group (not living with both parents $\mathrm{p}<0.001$, OR $0.69,95 \%$ CI 0.56 to 0.86 ; parent with diabetes $\mathrm{p}=0.033$; OR $0.71,95 \%$ CI 0.52 to $0.96, \mathrm{n}=742$ ). There was no relation between Townsend index and outpatient attendance rates.

No significant association was identified between medium term control as reflected in glycated haemoglobin (multiple of mean values) and levels of deprivation, parental diabetes, or single parent family structure.

\section{Discussion}

This study shows that children with diabetes living in the south west region of England appear to be neither socially advantaged nor disadvantaged as measured by the Townsend index. The large numbers of children with and without diabetes make it unlikely that any association of diabetes with social advantage of clinical significance really exists in this population.

The comparison using prevalence rather than incidence is more appropriate when the control children without diabetes may have moved in and out of the region. When only new cases arising in the geographical area (that is, the incidence) are compared, then children without diabetes moving into the region during the ascertainment period should be excluded. As they cannot be identified and removed from the control population the possibility of bias may exist.

In this study, there was a three year difference between the 1991 census and the ascertainment of the two populations of children with and without diabetes. It is unlikely that systematic differences in the degree of change in social circumstances over the intervening three years exist between the two groups. The study cannot control for any tendency for social circumstances to change in families with a child with diabetes. Compared with adults, diabetes in children is much less likely to affect the earning abilities of the major wage earner. In the UK, treatments and equipment are free; even home blood glucose monitors tend to be supplied free of charge. Disability living allowance and its forerunner are intended to help families cope with the negative impact of childhood diabetes, although not all families receive such help. In this study we did not find any association between having a parent with diabetes and the Townsend deprivation index.

The Townsend index uses four independent measures of social advantage. It has been developed and validated in UK populations. It has been suggested that the score is more relevant to children living in urban areas; families without a car in rural areas are likely to be more disadvantaged. These potential differences apply equally to both groups. With the caveats discussed, this study does not show any evidence of an association with social circumstances, either as cause or effect.

The lack of association between social advantage and diabetes has important implications for our understanding of causative factors, many of which are associated with social circumstances. For example, if lack of breast feeding is implicated in the aetiology, this would be expected to result in an increased rate of diabetes in socially disadvantaged children.

The great majority of children who were not with both parents lived apart from their fathers. The absence of one or both parents from the home is not only likely to result in lower self esteem and increased health, educational, and social difficulties in the child, ${ }^{16}$ but may also have an association with social disadvantage. It was possible to examine the association between social deprivation and family composition in the children in this study. Those not living with both parents had slightly, but significantly, higher Townsend indices. The use of logistic regression models is intended to allow examination of each factor separately. Associations with family composition but not Townsend index may therefore reflect the personal consequences for the child of the absence of one parent rather than the accompanying social disadvantage.

The relatively small numbers of children (45) who were $<5$ years of age at the time of the study makes generalisation of the findings to this age group difficult. It should be noted, however, that over a third of children in the study were $<5$ years at diagnosis. The stage when family and social circumstances are particularly important is arguably the first five years, and this is also a common age for diabetes to present.

There were higher admission rates with hypoglycaemia in children with social disadvantage but this association was not seen with 
family composition. Higher rates may indicate more episodes of severe hypoglycaemia or a greater reliance on the hospital when an episode does occur.

There was a strong association (OR 2.2) between hospital admission rates with poor diabetes control and the absence of one or both parents from the home but not with the Townsend index. This suggests that the child's family composition rather than social disadvantage may have been the important factor. The association (OR 2.1) between hospital admission rates with poor control in children having a parent with diabetes was similar in magnitude. However, the smaller numbers involved result in this being of borderline statistical significance. If this association is real, in the absence of association with Townsend index it would again suggest that family composition, rather than any associated social disadvantage, is important.

Despite these significant differences in indirect measures of diabetes control, there was no accompanying difference in glycated haemoglobin concentrations. A previous study of 43 children and adolescents used a direct measure of family stress. ${ }^{17}$ A positive association between glycated haemoglobin and high family stress levels was found, which was ameliorated by high levels of social support to mothers.

Clinic attendance rates similarly appeared to be adversely affected by the absence of one parent but not by social disadvantage as evidenced by the Townsend index. This could have important longer term implications, given the finding of an association between irregular clinic attendance and a higher rate of diabetes complications at follow up. ${ }^{18}$

Although there is a widely held impression that children with diabetes and an affected parent are likely to be poorly controlled this was not borne out entirely by this study in that medium term control was similar to other children. There was a suggestion, however, that other outcome variables such as rates of readmission for ketoacidosis or for stabilisation were higher. Clinic attendance rates were also lower.

In conclusion, children with diabetes living in the south west region of England were neither socially advantaged nor disadvantaged. However, socially deprived children were more likely to be admitted to hospital with hypoglycaemia. Children not living with both parents were slightly socially disadvantaged; they were more likely to be admitted to hospital with diabetes related problems and were less likely to attend outpatient appointments. Parental diabetes may have detrimental effects on some aspects of diabetes outcome.

1 Staines A, Bodansky HJ, McKinney PA, et al. Small area variation in the incidence of childhood insulin-dependent diabetes mellitus in Yorkshire, UK: links with overcrowding and population density. Int F Epidemiol 1997;26:1307-13.

2 Baumer JH, Hunt LP, Shield JPH. Audit of diabetes care by caseload. Arch Dis Child 1997;77:102-8.

3 Debono J, Johnson C, Betts P. Juvenile diabetes and social class [letter]. Lancet 1983;i:1113.

4 Tarn AC, Gorsuch AN, Spencer KM, Bottazzo GF, Lister J. Diabetes and social class [letter]. Lancet 1983;ii:631-2.

5 Stewart-Brown S, Haslum M, Butler N. Evidence of increasing prevalence of diabetes mellitus in childhood. BMF 1983;286:1855-7.

6 Siemiatycki J, Colle E, Campbell S, Dewar R, Aubert D, Belmonte M. Incidence of IDDM in Montreal by ethnic group and by social class and comparisons with ethnic groups living elsewhere. Diabetes 1988;37:1096-1102.

7 Patterson CC, Waugh NR. Urban/rural and deprivational differences in incidence and clustering of childhood diabetes in Scotland. Int f Epidemiol 1992;21:108-17.

8 Blom L, Dahlquist G, Nystrom L, Sandstrom A, Wall S. The Swedish childhood diabetes study-social and perinatal determinants for diabetes in childhood. Diabetologia 1989; 32:7-13.

9 Waugh NR. Insulin-dependent diabetes in a Scottish region: incidence and urban/rural differences. F Epidemiol Coтmunity Health 1986;40:240-3.

10 Crow YJ, Alberti KGMM, Parkin JM. Insulin dependent diabetes in childhood and material deprivation in Northern England, 1977-86. BMf 1991;303:158-60.

11 LaPorte, RE, Orchard TJ, Kuller LH, et al. The Pittsburgh insulin dependent diabetes registry. The relationship of insulin dependent diabetes mellitus incidence to social class. Am $\mathcal{F}$ Epidemiol 1981;114:379-84.

12 Carstairs V, Morris R. Deprivation and mortality: an alternative to social class? Community Medicine 1989;11: 210-19.

13 Phillimore P, Beattie A, Townsend P. Widening inequality of health in northern England, 1981-91. BM7 1994;308: $1125-8$.

14 Kirk CR, Savage DCL. The diabetic with a diabetic parent. Arch Dis Child 1985;60:572-86.

15 Kelly WF, Mahmood R, Turner S, Elliott K. Geographical mapping of diabetic patients from the deprived inner city shows less insulin therapy and more hyperglycaemia. Diabet Med 1994;11:344-8.

16 Tripp JH, Cockett M. Parents, parenting, and family breakdown. Arch Dis Child 1998;78:104-7.

17 Viner R, McGrath M, Trudinger P. Family stress and metabolic control in diabetes. Arch Dis Child 1996;74:418-21.

18 Jacobson AM, Hauser ST, Willett J, Wolfsdorf JI, Herman $\mathrm{L}$. Consequences of irregular versus continuous medical follow-up in children and adolescents with insulindependent diabetes mellitus. 7 Pediatr 1997;131:727-33. 